\title{
$M$-ACCRETIVE OPERATORS WITH $M$-DISPERSIVE RESOLVENTS ${ }^{1}$
}

\author{
RALPH DELAUBENFELS
}

\begin{abstract}
We characterize linear $m$-accretive operators with $m$-dispersive resolvents. $T$ is linear and $m$-accretive, with $(\lambda+T)^{-1} m$-dispersive, if and only if the sequence $\left\langle n ! \phi(\lambda+T)^{n+1} x\right\rangle_{n=0}^{\infty}$ equals the moments of a positive measure on the positive real line, for sufficiently many $\phi$ in $X^{*}, x$ in $X$.
\end{abstract}

Introduction. Classical analysis is often very useful in linear operator theory. In another paper, we show that $T$ is a spectral operator of scalar type when the sequence $\left\langle\phi\left(T^{n} x\right)\right\rangle_{n=0}^{\infty}$ equals the moments of a positive measure, for sufficiently many $\phi$ in $X^{*}, x$ in $X$. In this paper, we consider the sequence $\left\langle n ! \phi(\lambda+T)^{n+1} x\right\rangle_{n=0}^{\infty}$, for some positive $\lambda$, and again apply the classical analysis of the moment problem.

We say that a sequence of real numbers $\left\langle a_{n}\right\rangle_{n=0}^{\infty}$ satisfies a Stieltjes moment condition if $\sum \alpha_{k} \overline{\alpha_{j}} a_{k+j} \geqslant 0$ and $\sum \alpha_{k} \overline{\alpha_{j}} a_{k+j+1} \geqslant 0$, for all finite sequences $\left\langle\alpha_{k}\right\rangle$ of complex numbers. These conditions are satisfied if and only if there exists a positive measure $\mu$ so that

$$
a_{n}=\int_{0}^{\infty} t^{n} d \mu(t) \text { for all } n \quad(\text { see }[3])
$$

We will say $\left\langle a_{n}\right\rangle_{n=0}^{\infty}$ is Stieltjes if, in addition, $\sum_{n} \inf _{k \leqslant n}\left|a_{k}\right|^{-1 / 2 k}$ is infinite. When $\left\langle a_{n}\right\rangle_{n=0}^{\infty}$ is Stieltjes, the positive measure $\mu$ is unique.

If $T$ is a linear operator, then a vector $x$ is quasi-analytic for $T$ if $\sum_{n} \inf _{k \leqslant n}\left\|T^{k} x\right\|^{-1 / k}$ is infinite. $\mathscr{D}_{q}(T)$ is the set of all quasi-analytic vectors for $T$ (see [1]).

$T$ is $m$-accretive if it is densely defined and $(-T)$ generates a 1-parameter contraction semigroup. If the semigroup is positive, on a Banach lattice, $T$ is m-dispersive.

See [2] for other terminology in operator theory, such as accretive and core.

THEOREM. Suppose $X$ is a Banach lattice, $T$ is closed, densely defined and accretive, and there exists $\mathscr{D} \subseteq X^{+} \cap \mathscr{D}_{q}(T)$ such that $(\lambda+T)(\mathscr{D}) \subseteq \mathscr{D}$ and span $(\mathscr{D})$ is a core for $(\lambda+T)^{2}$, for some $\lambda>0$. Then $T$ is $m$-accretive, with $(\lambda+T)^{-1} m$-dispersive if and only if the sequence $\left\langle n ! \phi(\lambda+T)^{n+1} x\right\rangle_{n=0}^{\infty}$ satisfies a Stieltjes moment condition for all $\phi$ in $\left(X^{*}\right)^{+}, x$ in $\mathscr{D}$.

Received by the editors June 7, 1983 and, in revised form, October 21, 1983. Presented at 90 th annual meeting of the AMS, January 25-29, 1984.

1980 Mathematics Subject Classification. Primary 47B44.

${ }^{1}$ This paper is contained in the author's $\mathrm{Ph}$. D. thesis at the University of California, Berkeley. 
Proof. Suppose $T$ is $m$-accretive, with $(\lambda+T)^{-1} m$-dispersive.

We have, for all $\phi$ in $X^{*}, x$ in the domain of $T$, the resolvent formula

$$
n ! \phi(\lambda+T)^{n+1} x=\int_{0}^{x} s^{n} \phi\left(e^{-s(\lambda+T)^{-1}} x\right) d s .
$$

When $\phi \in\left(X^{*}\right)^{+}, x \in \mathscr{D}$, then $e^{-s(\lambda+T)^{-1}}$, the semigroup generated by $(\lambda+T)^{-1}$ is positive, so $\phi\left(e^{-s(\lambda+T)^{-1}} x\right) d s$ is a positive measure. Thus, $\left\langle n ! \phi(\lambda+T)^{n+1} x\right\rangle_{n=0}^{x^{x}}$ satisfies a Stieltjes moment condition. (Note that the density of the domain of $T$ was not needed here.)

Conversely, suppose $\left\langle n ! \phi(\lambda+T)^{n+1} x\right\rangle_{n=0}^{\infty}$ satisfies a Stieltjes moment condition, for all $\phi$ in $\left(X^{*}\right)^{+}, x$ in $\mathscr{D}$.

Since $x \in \mathscr{D}_{q}(T)$, the sequence is Stieltjes. Thus, if $\phi \in\left(X^{*}\right)^{+}, x \in \mathscr{D}$, there exists a unique positive measure $\mu_{\phi . x}$ such that

$$
n ! \phi(\lambda+T)^{n+1} x=\int_{0}^{\infty} t^{n} d \mu_{\phi . . x}(t) \text { for all } n .
$$

Note that

$$
\begin{aligned}
\int_{0}^{\infty} t^{n} \int_{t}^{\infty} d \mu_{\phi, x}(s) d t & =\int_{0}^{\infty} \int_{0}^{s} t^{n} d t d \mu_{\phi . x}(s) \\
& =\int_{0}^{\infty} \frac{s^{n+1}}{n+1} d \mu_{\phi . x}(s)=n ! \phi(\lambda+T)^{n+2} x \\
& =\int_{0}^{\infty} t^{n} d \mu_{\phi .(\lambda+T) . x}(t) .
\end{aligned}
$$

Since the positive measure in (1) is unique, we have

$$
\int_{t}^{\infty} d \mu_{\phi . x}(s) d t=d \mu_{\phi .(\lambda+T) x}(t), \text { for all } \phi \text { in }\left(X^{*}\right)^{+}, x \in \mathscr{D} .
$$

This implies that $d \mu_{\phi,(\lambda+T)^{2} x}(t) / d t$ exists and is a continuous function of $t$. For each $t$, define $F(t):(\lambda+T)^{2}(\mathscr{D}) \rightarrow X^{* *}$ by

$$
[F(t) y](\phi) \equiv \frac{d \mu_{\phi . y}(t)}{d t}
$$

if $\phi$ is positive. For arbitrary $\phi \in X^{*}$, there exist positive $\phi_{1}, \phi_{2}, \phi_{3}, \phi_{4}$, with $\phi_{1}$ orthogonal to $\phi_{2}, \phi_{3}$ orthogonal to $\phi_{4}$ and $\phi=\left(\phi_{1}-\phi_{2}\right)+i\left(\phi_{3}-\phi_{4}\right)$. Then

$$
[F(t) y] \phi \equiv[F(t) y] \phi_{1}-[F(t) y] \phi_{2}+i\left([F(t) y] \phi_{3}-[F(t) y] \phi_{4}\right) \text {. }
$$

To see that $F(t) y \in X^{* *}$, note that if $\phi \in\left(X^{*}\right)^{+}$,

$$
|F(t)(\lambda+T) x(\phi)|=\int_{t}^{x} d \mu_{\phi, . x}(s) \leqslant \int_{0}^{\infty} d \mu_{\phi, . x}(s)=\phi(\lambda+T) x .
$$

Since $F(t)(\lambda+T) x$ is positive, this implies that $\|F(t)(\lambda+T) x\| \leqslant\|(\lambda+T) x\|$. Thus $\|F(t)\| \leqslant 1$, for all $t \geqslant 0$.

Note that $F(0)(\lambda+T) x=\int_{0}^{\infty} F(s) x d s=(\lambda+T) x$. Thus $F(0)=I$. Collecting all the facts about $F(t)$ : For all $y \in(\lambda+T)^{2}(\mathscr{D}), \phi \in\left(X^{*}\right)^{+}$,

$$
[F(t) y](\phi) \text { is positive continuous function of } t \text {, }
$$

$$
F(0)=I \text {, }
$$




$$
\begin{aligned}
n ! \phi(\lambda+T)^{n+1} y & =\int_{0}^{\infty} t^{n}[F(t) y](\phi) d t, \\
\int_{t}^{\infty}[F(s) y](\phi) d s & =[F(t)(\lambda+T) y](\phi) .
\end{aligned}
$$

To show that $T$ is $m$-accretive, we need to show that the range of $(\lambda+T)$ is dense. First, we will show that the range of $(\lambda+T)^{3}$ is dense in the range of $(\lambda+T)^{2}$, written $R(\lambda+T)^{2}$.

So suppose $\phi \in\left[R(\lambda+T)^{3}\right]^{\perp}$, the annihilator of $R(\lambda+T)^{3}$. There exist positive $\phi_{1}, \phi_{2}, \phi_{3}, \phi_{4}$ with $\phi_{1}$ orthogonal to $\phi_{2}, \phi_{3}$ orthogonal to $\phi_{4}$, so that $\phi=\left(\phi_{1}-\right.$ $\left.\phi_{2}\right)+i\left(\phi_{3}-\phi_{4}\right)$.

Suppose $y \in(\lambda+T)^{2}(\mathscr{D})$. Then, for all $n, 0=n ! \phi(\lambda+T)^{n+1} y$. By (6), this implies that

$$
\int_{0}^{\infty} t^{n}[F(t) y]\left(\phi_{1}\right) d t=\int_{0}^{\infty} t^{n}[F(t) y]\left(\phi_{2}\right) d t .
$$

Since $[F(t) y]\left(\phi_{i}\right)$ is positive, for $i=1,2$, the uniqueness of the positive measure in (1) implies that $[F(t) y] \phi_{1} d t=[F(t) y] \phi_{2} d t$. Since $F(t)$ is continuous, this implies that $[F(t) y] \phi_{1}=[F(t) y] \phi_{2}$; in particular,

$$
\phi_{1}(y)=[F(0) y] \phi_{1}=[F(0) y] \phi_{2}=\phi_{2}(y) .
$$

By an identical argument, $\phi_{3}(y)=\phi_{4}(y)$. Thus $\phi(y)=0$.

Since the span of $\mathscr{D}$ is a core for $(\lambda+T)^{2},(\lambda+T)^{2}(\mathscr{D})$ is total in $R(\lambda+T)^{2}$. Since $\phi$ annihilates the total set $(\lambda+T)^{2}(\mathscr{D}), \phi \in\left[R(\lambda+T)^{2}\right]^{\perp}$.

Thus, since $T$ is closed, $R(\lambda+T)^{2}=R(\lambda+T)^{3}$.

Since $T$ is accretive, this implies that $T$ is $m$-accretive, on $R(\lambda+T)^{2}$. This implies that $(\lambda+T)^{-1}$ is a bounded operator on $R(\lambda+T)^{2}$. Thus, if $x$ is in the domain of $T$, then $x=(\lambda+T)^{-2}(\lambda+T)^{2} x$ is in $R(\lambda+T)^{2}$. Since the domain of $T$ is dense, and $T$ is closed, $R(\lambda+T)^{2}$, and therefore $R(\lambda+T)$, equals $X$. Thus $T$ is $m$-accretive.

To show that $T$ has an $m$-dispersive resolvent, we will extend $F(t)$ to all of $\mathscr{D}$, and show that it equals the semigroup generated by $(\lambda+T)^{-1}$.

Differentiating both sides of (7) gives

$$
-F(t) y=F^{\prime}(t)(\lambda+T) y \text { for all } y \in(\lambda+T)^{2}(\mathscr{D}) .
$$

This implies that $F(t) y=e^{-t(\lambda+T)^{-1}} y$ (the semigroup exists, because $(\lambda+T)^{-1}$ is bounded). Thus $F(t)(\lambda+T)^{2} x$ is a $C^{\infty}$ function of $t$, for all $x$ in $\mathscr{D}$.

By (2), this implies that $d \mu_{\phi, x}(t) / d t$ exists, and is a $C^{\infty}$ function of $t$, for all $x$ in $\mathscr{D}, \phi$ in $\left(X^{*}\right)^{+}$.

Define $G(t): \mathscr{D} \rightarrow X^{* *}$ exactly as $F(t)$ was defined in (3). Equations (4)-(8) all hold for $G(t)$.

Since $e^{-t(\lambda+T)-1} x$ (equal to $G(t) x$ ) is positive, for all $x$ in $\mathscr{D}$, and $\mathscr{D}$ equals $X^{+}$, $e^{-t(\lambda+T)^{-1}}$ is positive, so that $(\lambda+T)^{-1}$ is $m$-dispersive, concluding the theorem.

The hypotheses are clearly satisfied when $T$ is bounded and accretive (letting $\left.\mathscr{D}=X^{+}\right)$. Note that $T$ is automatically $m$-accretive. 
Corollary. Suppose $X$ is a Banach lattice and $T$ is bounded and accretive. Then $(\lambda+T)^{-1}$ is $m$-dispersive if and only if $\left\langle n ! \phi(\lambda+T)^{n+1} x\right\rangle_{n=0}^{\infty}$ satisfies a Stieltjes moment condition for all $\phi$ in $\left(X^{*}\right)^{+}, x$ in $X^{+}$.

\section{REFERENCES}

1. P. R. Chernoff, Quasi-analytic vectors and quasi-analytic functions, Bull. Amer. Math. Soc. 81 (1975), 637-646.

2. T. Kato, Perturbation theory for linear operators, Springer-Verlag, Berlin and New York, 1966.

3. J. A. Shohat and J. D. Tamarkin, The problem of moments, Math. Surveys, vol. 1, Amer. Math. Soc., Providence, R. I., 1943.

Department of Mathematics, Dartmouth College, Hanover, New Hampshire 03755

Current address: Department of Mathematical Sciences, The University of Tulsa, 600 South College Avenue, Tulsa, Oklahoma 74104 